\title{
Programmable quasi-direct space-to-time pulse shaper with active wavefront correction
}

\author{
Omel Mendoza-Yero, ${ }^{1, *}$ Vincent Loriot, ${ }^{2,3}$ Jorge Pérez-Vizcaíno, ${ }^{1}$ Gladys Mínguez-Vega, ${ }^{1}$ \\ Jesús Lancis, ${ }^{1}$ Rebeca de Nalda, ${ }^{2}$ and Luis Bañares ${ }^{3}$ \\ ${ }^{1}$ Institut de Noves Tecnologies de la Imatge (INIT), Universitat Jaume I, Castelló E 12080, Spain \\ ${ }^{2}$ Instituto de Química Física Rocasolano, Consejo Superior de Investigaciones Científicas, Madrid 28006, Spain \\ ${ }^{3}$ Facultad de Ciencias Químicas, Universidad Complutense de Madrid, Madrid 28040, Spain \\ *Corresponding author: omendoza@fca.uji.es
}

Received October 18, 2012; revised November 8, 2012; accepted November 8, 2012; posted November 9, 2012 (Doc. ID 178289); published December 5, 2012

\begin{abstract}
We experimentally demonstrate an extremely compact and programmable pulse shaper composed of a single phase mask encoded into a spatial light modulator. Its principle of operation is similar to the previously theoretically introduced quasi-direct space-to-time pulse shaper [Opt. Express 16, 16993 (2008)], which is based on diffractive optics. The proposed pulse shaper exhibits not only real-time temporal modulation, but also high-efficiency output pulses thanks to an active correction of the wavefront aberrations. (c) 2012 Optical Society of America

OCIS codes: $320.5540,050.1970,220.1000$.
\end{abstract}

Today, femtosecond pulse shaping is a widespread technology. User-defined temporal waveforms with control of phase, amplitude, and polarization are required in many applications, such as high-field laser-matter interactions, photonic processing of telecom signals, and nonlinear microscopy. Pulse shaping is usually performed through a space-to-time transformation, where spatial masking of an optical beam causes its temporal modulation. In the Fourier-transform pulse shaping technique, the complex mask is placed at the Fourier plane of a zero-dispersion grating device where the optical frequency spectrum is spatially dispersed [1].

In a different approach, if a spatial mask is placed in the input plane of a generalized spectrometer, a direct (for a diffractive grating) or quasi-direct [for a diffractive lens (DL)] space-to-time mapping of the input spatial mask into the output temporal waveform is obtained. The advantages of these approaches are the compactness of the setups and the saving of time, as the Fouriertransform computation of the spatial patterns is not needed. In this context, the direct space-to-time (DST) [2] and the quasi-direct space-to-time (QDST) [3, 4$]$ pulse shapers have demonstrated their utility for highrepetition-rate wavelength-division-multiplexed systems [5], the generation of millimeter waves [ $\underline{6}$ ], the generation of fractal light pulses [7], and the study of molecular alignment [8], and also in the spectral domain to obtain bandpass optical filters $[9,10]$.

Compact, dynamic, user-friendly, and fast-operation devices demanded by nonexpert pulse-shaper users have inspired researchers to look for simple and compact designs. In this context, DST pulse shapers have been recently reduced to a phase-only spatial light modulator (SLM) together with a refractive lens to create a very innovative device [11]. However, high-power Ti:sapphire laser systems suffer from wavefront aberrations due to imperfections in the optical components, and pumpinduced thermal distortions in the amplifiers. Although thermal distortions can be reduced by cooling the Ti:sapphire crystal, an accurate correction of wavefront aberrations needs a more versatile approach usually based on adaptive optics $[\underline{12}, \underline{13}]$.
In this Letter, we show that the QDST pulse shaper can be reduced to a simple phase mask. As far as we know, this is the first proposal of a single diffractive element pulse shaper. In this proposal the entire size of the pulse shaper is very small; there is almost no need for optical alignment, and obviously no extra refractive focusing element is required. From an experimental point of view, the abovementioned phase mask is implemented into a phase-only SLM, exhibiting in this way an additional advantage, its programmable performance. Furthermore, we demonstrate that an appropriate correction of the wavefront aberrations improves the pulse features at the output plane of the shaper. At this point, by using experimental data collected with a commercial Shark-Hartmman wavefront sensor (WS), the aberrations were mostly corrected with the SLM. In the present implementation of the QDST pulse shaper, both the corresponding phase mask and the wavefront correction were accomplished with the same device, the SLM. In order to measure the temporal response of the shaper, an intensity cross correlation [14], with a nonlinear crystal, between the shaped and the reference ultrashort light pulse has been carried out.

In Fig. 1 a schematic diagram of the optical setup is shown. For the experiment we use a Ti:sapphire

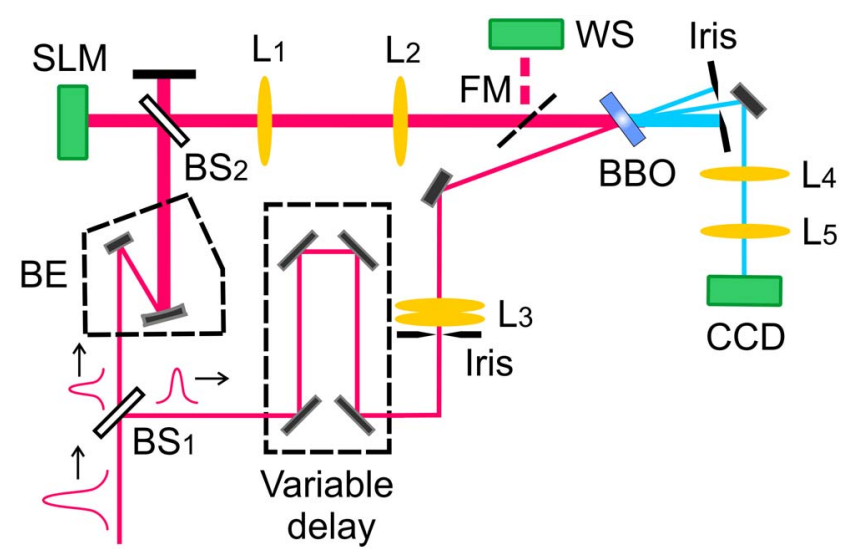

Fig. 1. (Color online) Schematic of the intensity crosscorrelation setup to measure the temporal profile of light pulses. 
femtosecond laser that emits pulses of about $30 \mathrm{fs}$ intensity FWHM at $1 \mathrm{kHz}$ of repetition rate, centered at $\lambda_{0}=$ $800 \mathrm{~nm}$ with $40 \mathrm{~nm}$ of spectral bandwidth. At the entrance of the interferometer, the light coming from the ultrashort laser source is split with a pellicle beam splitter $\left(\mathrm{BS}_{1}\right)$ into two pulses: the reference pulse and the shaped pulse. The reference pulse is a replica of the initial ultrashort pulse, whereas the shaped pulse has the desired temporal profile. The latter is first expanded via $4 \times$ all-mirror beam expander (BE) and sent, using a second beam splitter $\left(\mathrm{BS}_{2}\right)$, to the phase-only SLM $(1920 \times 1080$ pixel resolution, and $8 \mu \mathrm{m}$ pixel pitch $)$, where the phase mask of the QDST pulse shaper is encoded.

In previous versions $[\underline{3}, 8]$, the QDST pulse shaper was composed of two separate elements: a DL and a complex mask facing each other. To construct a single phase mask, both the complex mask transmittance and the DL must be encoded onto the phase-only SLM. The complex mask transmittance is written as $|t(r)| \exp \left(i \varphi_{M}\right)$, where $|t(r)|$ is the amplitude and $\varphi_{M}$ is the phase of the function. To do that, we follow a procedure reported in [15]. That is, for each pixel of the SLM, the encoded phase mask is obtained by multiplying the amplitude of the complex mask by the phase of the DL. Thus, the phase transmission function of the phase-only SLM is $T(r)=\exp \left[i|t(r)|\left(\varphi_{M}+\Phi_{\mathrm{DL}}\right)\right]$, where $\Phi_{\mathrm{DL}}=$ $\pi r^{2} /\left(\lambda_{0} Z_{0}\right)$ is the phase introduced by the DL, and $Z_{0}$ denotes its focal length for $\lambda_{0}$. The amplitude of the complex mask transmittance must be defined within the range $[0,1]$, whereas the total phase between $[-\pi, \pi]$. From the previous procedure it is apparent that as the depth of the phase distribution changes with $|t(r)|$, the diffraction efficiency of the phase mask is spatially modified.

The output of the QDST pulse shaper is located at the plane $z=Z_{0}$, in the close vicinity (by few micrometers) of the focal spot of the DL. In our optical setup, the plane $z=Z_{0}$ was imaged onto the plane of the nonlinear crystal with the help of a telescope composed of two lenses $\left(\mathrm{L}_{1}\right.$ and $\mathrm{L}_{2}$ ) of equal focal length $(200 \mathrm{~mm})$. The temporal amplitude of the shaped pulse is approximately given by the convolution of the initial ultrashort pulse with the transmittance of the complex mask in $r^{2}$, previously transformed into the time domain by the conversion constant $\beta=1 /\left(2 c Z_{0}\right)$, where $c$ is the velocity of light in vacuum [3]. Furthermore, the temporal window of the shaper $\Lambda$ is assessed by the expression $\Lambda=r_{0}^{2} \beta, r_{0}=$ $4.3 \mathrm{~mm}$ being the radial extension of the phase mask.

The shaped and reference pulses are spatially and temporally overlapped into a $\beta$ - $\mathrm{BaB}_{2} \mathrm{O}_{4}$ (BBO) type I crystal of $70 \mu \mathrm{m}$ thickness. In our case, the crystal is thin enough to ensure a phase-matching process without loss of spectral components. The reference pulse is focused by means of a pair of refractive lenses $\left(\mathrm{L}_{3}\right)$, with an equivalent focal length of $250 \mathrm{~mm}$. Since the length of bulk material is approximately the same in both arms, the effects of group-velocity dispersion were pre-compensated with a prism pair at the postcompression stage of the laser.

The delay line shown in Fig. 1 allows us to vary the path length of the reference pulse. By recording the intensity of the central second-harmonic (SH) signal for each position of the delay line, the approximated intensity profile (temporally stretched by the convolution with the reference pulse) of the shaped pulse is determined. In order to select the SH signal generated only in the close vicinity of the DL focus, we formed a $2 \times$ magnified image of the $\mathrm{BBO}$ crystal plane onto a CCD camera with a telescope composed of lenses $\mathrm{L}_{4}$ and $\mathrm{L}_{5}$ of focal lengths 100 and $200 \mathrm{~mm}$, respectively.

In practice, the above measurements are affected by wavefront aberrations. That is, while moving the delay line to temporally scan the shaped pulse with respect to the reference one, the wavefront aberrations deteriorate the irradiance distribution at the $\mathrm{BBO}$ crystal plane. Here, the propagation time difference (PTD) among pulses coming from the cross section of the DL describes the temporal overlapping process in the BBO crystal. For each position of the delay line, the irradiance distribution at the $\mathrm{BBO}$ crystal plane is determined by the coherent interference of pulses diffracted from ring zones of the DL, with the reference pulse. These zones of equal area correspond to geometrical rings of the DL from which the PTD of diffracted pulses is equal to (or less than) the temporal pulse width of the reference pulse. The larger and thinner the rings, the smaller the focal spot size of the DL, and consequently the effects of wavefront aberrations become more and more critical.

In Fig. 2(a) we show the spatial shape of the central SH signal in the case of the outermost ring zone of the DL (achieved at the corresponding position of the delay line). From Fig. 2(a) it is clear that the expected Bessellike pattern is completely lost because of the presence of aberrations. The above point was confirmed with an active correction of the wavefront aberrations at the BBO crystal plane; see Fig. 2(b).

For this purpose, we deviated the shaped pulse before the BBO crystal using a flip mirror (FM) (see Fig. 1), and sent it toward a commercial Shark-Harmman WS, where the spatial phase $\varphi_{\mathrm{SP}}$ at the SLM plane was measured. Hence, the phase transmission function of the phaseonly SLM is finally rewritten in the form $T(r)=$ $\exp \left[i|t(r)|\left(\varphi_{M}+\Phi_{\mathrm{DL}}-\varphi_{\mathrm{SP}}\right)\right]$. In this way, the SLM is not only used to encode the QDST phase mask, but also to correct for wavefront distortions. The comparison between Figs. 2(a) and 2(b) allows us to see the improvement of the focal distribution after wavefront correction.

The finite number of pixels of the SLM causes the efficiency of the encoded DLs to depend on the radial coordinate. This unwanted effect originates a modulation in the temporal response of the QDST pulse shaper,
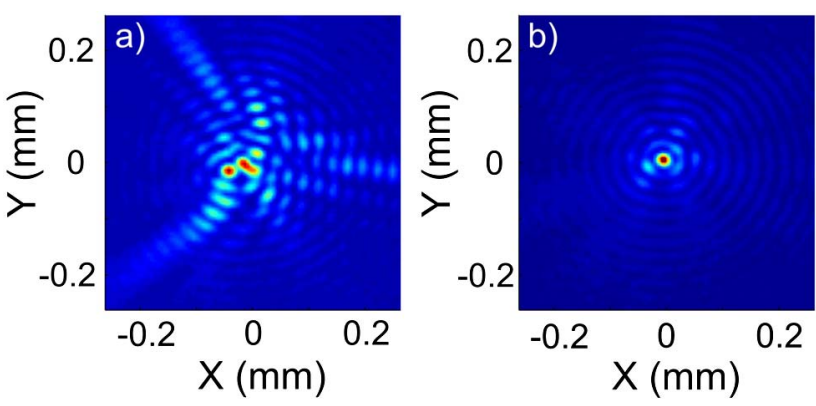

Fig. 2. (Color online) SH irradiance patterns at the BBO crystal plane for the outermost ring zone of the DL, without (a) and with (b) wavefront correction. 


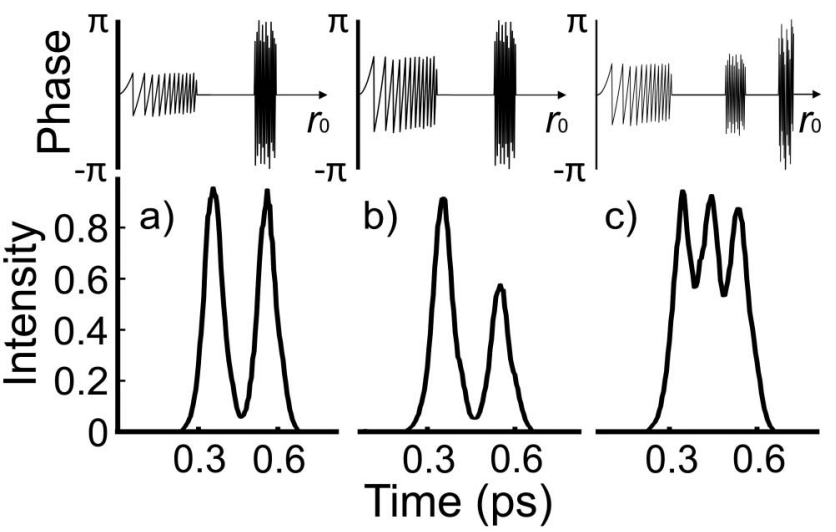

Fig. 3. Intensity cross-correlation measurements of temporal profiles synthesized by the QDST pulse shaper (bottom). Example of a radial phase profile of corresponding masks (top).

decreasing the peak intensity of the shaped pulses coming from the outer zones of the encoded DL. For longer focal lengths the above-mentioned dependence can be turned off, but unfortunately in these cases the temporal window of the QDST pulse shaper is also reduced. In particular, we encode a DL with focal length of $135 \mathrm{~mm}$, which corresponds to a temporal window of about $230 \mathrm{fs}$. In this case, the phase of the outermost ring of the DL is encoded with only two phase levels. This reduces the focalization efficiency to about 40\% [16]. Therefore, to guarantee a constant response over the whole temporal window of the QDST pulse shaper the amplitude of the complex mask should change radially to compensate for the mentioned efficiency loss (see mask profiles at top part of Fig. 3)

In Fig. 3 , three different examples of temporal profiles obtained with the QDST pulse shaper are shown. The first case [see Fig. 3(a)] is a sequence of two pulses of equal peak intensity, delayed 200 fs between them. The temporal width of the shaped pulses, considering their convolution with the reference pulse, is about 80 fs intensity FWHM. The second example shown in Fig. 3(b) corresponds to the same temporal profile, but different relative peak intensity between pulses. Finally, the third case is a sequence of three pulses with slowly decreasing peak intensity; see Fig. 3(c). For examples shown in Figs. 3(a)-3(c), the theoretical overall diffraction efficiencies are $2.70 \%, 2.48 \%$, and $2.67 \%$, respectively. Notice that the limited spatial size of the SLM and the temporal width of the initial ultrashort pulse establish the maximum number of pulses that can be synthesized within the available temporal window; see Fig. 3(c).

For certain applications, an array of phase masks can also be possible, giving the extra possibility of parallel spatiotemporal and dynamical processing. In all applications, before implementing the QDST pulse shaper, particular attention should be paid to the adequate correction of the wavefront aberrations. The temporal window of the programmable QDST pulse shaper could be also increased in the near future when technological advances allow for SLM with larger working area as well as smaller pixel pitch. This is not the case for static phase masks, where the available techniques (e.g., photolithography) can achieve several centimeters for the mask size while keeping high spatial resolution.

This research was funded by the Spanish Ministerio de Cienciae Innovación and the GeneralitatValenciana through Consolider Programme (SAUUL CSD2007-00013), and Prometeo Excellence Programme (PROMETEO/2012/021), and projects FIS2010-15746, P11B2010-26, and CTQ200802578. We also thank the European network ITN FASTQUAST (PITN-GA-2008-214962). Authors are also very grateful to the SCIC of the Universitat Jaume I for the use of the femtosecond laser.

\section{References}

1. A. M. Weiner, Opt. Commun. 284, 3669 (2011).

2. D. E. Leaird and A. M. Weiner, Opt. Lett. 24, 853 (1999).

3. G. Mínguez-Vega, O. Mendoza-Yero, J. Lancis, R. Gisbert, and P. Andrés, Opt. Express 16, 16993 (2008).

4. V. Loriot, O. Mendoza-Yero, G. Mínguez-Vega, L. Bañares, and R. de Nalda, IEEE Photon. Technol. Lett. 24, 273 (2012).

5. D. E. Leaird, A. M. Weiner, S. Shen, A. Sugita, S. Kamei, M. Ishii, and K. Okamoto, Opt. Quant. Electron. 33, 811 (2001).

6. J. D. McKinney, D. E. Leaird, and A. M. Weiner, Opt. Lett. 27, 1345 (2002).

7. O. Mendoza-Yero, B. Alonso, G. Mínguez-Vega, Í. J. Sola, J. Lancis, and J. A. Monsoriu, Opt. Lett. 37, 1145 (2012).

8. O. Mendoza-Yero, G. Mínguez-Vega, J. Lancis, and V. Climent, Opt. Lett. 35, 535 (2010).

9. G. Mínguez-Vega, V. R. Supradeepa, O. Mendoza-Yero, and A. M. Weiner, Opt. Lett. 35, 2406 (2010).

10. G. Mínguez-Vega, O. Mendoza-Yero, E. Tajahuerce, J. Lancis, and P. Andrés, IEEE Photon. Technol. Lett. 21, 347 (2009).

11. T. Mansuryan, M. Kalashyan, J. Lhermite, E. Suran, V. Kermene, A. Barthelemy, and F. Louradour, Opt. Lett. 36, 1635 (2011).

12. H. Baumhacker, G. Pretzler, K. J. Witte, M. Hegelich, M. Kaluza, S. Karsch, A. Kudryashov, V. Samarkin, and A. Roukossouev, Opt. Lett. 27, 1570 (2002).

13. J. M. Bueno, B. Vohnsen, L. Roso, and P. Artal, Appl. Opt. 48, 770 (2009).

14. J. Trull, O. Jedrkiewicz, P. Di Trapani, A. Matijošius, A. Varanavičius, G. Valiulis, R. Danielius, E. Kucinskas, A. Piskarskas, and S. Trillo, Phys. Rev. E 69, 026607 (2004).

15. J. A. Davis, D. M. Cottrell, J. Campos, M. J. Yzuel, and I. Moreno, Appl. Opt. 38, 5004 (1999).

16. S. Sinzinger and J. Jahns, Microoptics (Wiley VCH, 2003). 